\title{
Searching for serial refreshing in working memory: Using response times to track the content of the focus of attention over time
}

\author{
Evie Vergauwe $^{1}$ - Kyle O. Hardman ${ }^{1}$ - Jeffrey N. Rouder ${ }^{1}$ - Emily Roemer ${ }^{2}$. \\ Sara McAllaster $^{3} \cdot$ Nelson Cowan $^{1}$
}

Published online: 11 April 2016

(C) Psychonomic Society, Inc. 2016

\begin{abstract}
One popular idea is that, to support the maintenance of a set of elements over brief periods of time, the focus of attention rotates among the different elements, thereby serially refreshing the content of working memory (WM). In the research reported here, probe letters were presented between tobe-remembered letters, and response times to these probes were used to infer the status of the different items in WM. If the focus of attention cycles from one item to the next, its content should be different at different points in time, and this should be reflected in a change in the response time patterns over time. Across a set of four experiments, we demonstrated a striking pattern of invariance in the response time patterns over time, suggesting either that the content of the focus of attention did not change over time or that response times cannot be used to infer the content of the focus of attention. We discuss how this pattern constrains models of WM, attention, and human information processing.
\end{abstract}

Keywords Working memory · Focus of attention ·

Refreshing $\cdot$ Serial refreshing

Electronic supplementary material The online version of this article (doi:10.3758/s13423-016-1038-1) contains supplementary material, which is available to authorized users.

Evie Vergauwe

vergauwee@missouri.edu

1 Department of Psychological Sciences, University of Missouri, Columbia, MI, USA

2 University of Rochester, Rochester, NY, USA

3 Chapman University, Orange, CA, USA
People must often maintain a set of elements active in mind over brief periods of time. This information is purportedly stored in working memory (WM). One proposed mechanism to keep information active in WM is refreshing. In contrast to covert or overt verbal rehearsal, refreshing is assumed to be a domain-general mechanism that operates by bringing WM representations into the focus of attention (Barrouillet \& Camos, 2012; Cowan, 1995; Higgins \& Johnson, 2009). The act of refreshing presumably results in memory representations being reactivated, which, in turn, protects the information from being forgotten.

Though considerable research in recent years has been devoted to the process of refreshing (e.g., Camos \& Portrat, 2015; Loaiza, Duperreault, Rhodes, \& McCabe, 2015; Souza, Rerko, \& Oberauer, 2015; Vergauwe \& Cowan, 2015), little is currently known about how refreshing operates to support the maintenance of a set of elements in WM. In the present study, we tested the strong hypothesis that refreshing operates serially, with the focus of attention cycling from one item to the next (e.g., Barrouillet \& Camos, 2012; Cowan, 2011; McCabe, 2008; Nee \& Jonides, 2013; Vergauwe, Camos, \& Barrouillet, 2014).

Evidence for a focus of attention in WM that is limited to one element at a time has come from studies showing that the element last processed has a privileged status of accessibility in WM, relative to other to-be-remembered elements. For example, in an item recognition task in which a list of items is followed by a probe to be judged as being either present in or absent from the list, response times (RTs) are faster to the last item than to any other item of the list (e.g., Burrows \& Okada, 1971; McElree \& Dosher, 1989; Nee \& Jonides, 2008; Oztekin, Dvachi, \& McElree, 2010); the last item of the list is accessed at a faster rate (see McElree, 2006, for a review); and distinct brain regions are involved in judging the last item of the list (e.g., Nee \& Jonides, 2008; Oztekin et al., 2010). 
The RT benefit for the last item in WM may be leveraged to assess whether serial refreshing is taking place. The logic is as follows: When refreshing happens, the last-presented item is replaced in the focus of attention by another list item. This other item will consequently now have the focus-of-attention benefit, if probed; the last-presented item, which was replaced, presumably will no longer have the focus-of-attention benefit. Refreshing thus should attenuate the last-presented-item benefit, and this attenuation can be assessed as an indirect index of serial refreshing.

\section{Overview of the study}

To test theoretical assumptions about refreshing, we created the probe-span task. In four experiments, short series of red letters were presented for subsequent recall, and black probe letters were presented between these memory items, with each probe to be judged as being present in or absent from the list presented so far, as quickly as possible (Fig. 1). We manipulated the delay between each studied item and the subsequent probe. If the delay before the probe is very short, then we would expect refreshing not yet to have occurred and the last item to remain in the focus of attention. In this case, responses to the last-presented item should be speeded. If the delay is long, however, then according to common assumptions, refreshing should have occurred; the most recently refreshed item would be assumed to be in the focus of attention, but its serial position should vary from trial to trial. In this case there should be no advantage, and responses to the lastpresented item should not be speeded. Of course, for the approach to work, the short and long delays would need to be chosen carefully to test the common assumptions. These issues are addressed subsequently. In summary, on the basis of assumptions stemming from the studies reviewed above, the serial-refreshing hypothesis predicts a specific interaction pattern between serial position and the duration of the delay before the probe, in which the last-presented item should be speeded only for short delays between the most recent memory item and the following probe.

Contrary to our expectations, we observed that participants were fastest to respond to the last-presented memory item at all probe delays. In fact, the duration of the delay before the probe did not affect the serial-position function at all. This invariance of the serial-position curves across time was replicated in three additional experiments that aimed to create optimal conditions to detect serial refreshing, by using (1) in Experiments $2-4$, probe delays similar to the durations allowed for refreshing in studies that have provided evidence of it (Barrouillet \& Camos, 2012); (2) in Experiments 3 and 4, a restricted set of phonologically similar letters as memoranda, inasmuch as people strategically favor refreshing over speechbased rehearsal for such materials (Camos, Mora, \& Oberauer,
2011); and (3) in Experiment 4, memoranda shortened from 1, 000 to $500 \mathrm{~ms}$, to ensure that refreshing could not occur during the latter part of the presentation time (cf. Oberauer \& Lewandowsky, 2011).

\section{Method}

\section{Participants}

The participants were undergraduate students at the University of Missouri-Columbia and were paid $\$ 15$ for their participation or received course credit. All were native speakers of English and had normal or correctedto-normal vision. Experiments 1 through 4, respectively, included 40 ( 24 female, 16 male), 60 (36 female, 24 male), 40 (21 female, 19 male), and 40 (15 female, 25 male) participants.

\section{Materials and procedure}

The probe-span task was administered using the E-Prime software (Psychology Software Tools). Participants were asked to watch carefully and memorize series of four red letters presented sequentially on screen. In Experiments 1 and 2, all consonants, excluding $\mathrm{Y}$, were used as the stimuli. In Experiments 3 and 4, a pool of eight phonologically similar consonants were used as the stimuli: B, C, D, G, P, T, V, and Z. In all experiments, the different consonants were used approximately equally often, and no consonant was repeated within a series. These red letters were presented at the center of the screen in 48 -point Courier New font $\left(\sim 2.29^{\circ}\right.$ of visual angle). Stimuli were presented on a standard CRT monitor, and participants sat at a comfortable distance from the screen $(\sim 50 \mathrm{~cm})$.

Each series began by a fixation cross, centrally displayed on screen for $750 \mathrm{~ms}$. This fixation signal was replaced by the first red letter. Red letters were presented for either 1,000 ms (Exps. 1-3) or $500 \mathrm{~ms}$ (Exp. 4). At the end of each series, an empty rectangle on screen prompted participants to recall the four red letters of that series in their order of appearance by typing them on the keyboard. Participants were encouraged to fill in unknown letters with a guess. All entered letters appeared in the box, from left to right. Participants pressed Enter to end the recall response, and initiated the next series by pressing a button on the button box after recall.

After each red memory item, one black letter (probe) was presented in the center of the screen in 24-point Courier New font $\left(\sim 1.15^{\circ}\right.$ of visual angle). Participants were instructed to decide whether or not this black letter corresponded to one of the red letters they were maintaining on the current trial. This judgment was made by pressing the rightmost button of the button box when the black letter corresponded to one of the 
a

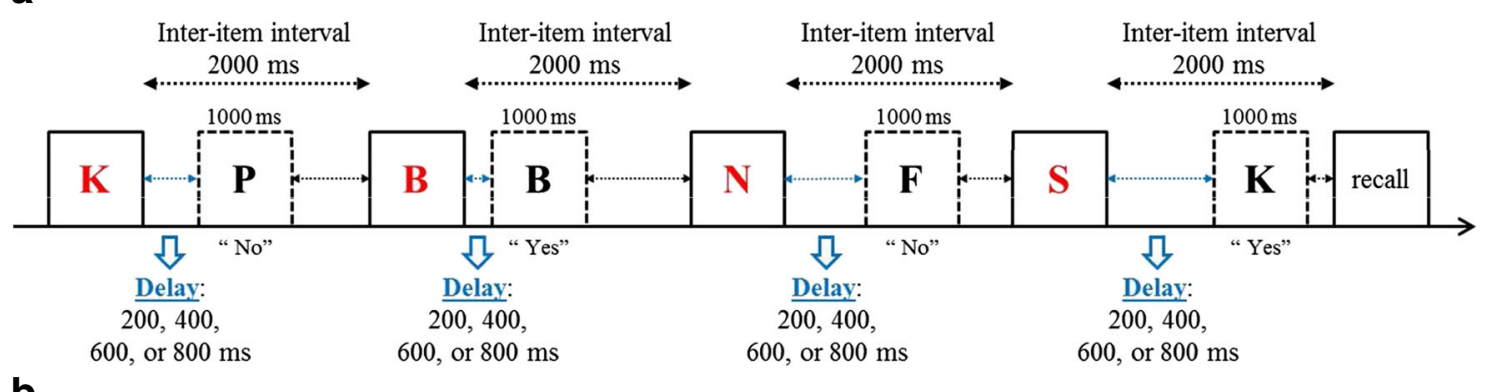

b

\begin{tabular}{lc|c|c}
\hline Memory material & Delay before probe & $\begin{array}{c}\text { Presentation duration } \\
\text { memory items }\end{array}$ \\
\hline$\underline{\text { Experiment 1 }}$ & Regular letters & $100,200,400 \mathrm{~ms}$ & $1000 \mathrm{~ms} /$ item \\
\hline$\underline{\text { Experiment 3 }}$ & $\begin{array}{c}\text { Phonologically } \\
\text { similar letters }\end{array}$ & $200,400,600,800 \mathrm{~ms}$ & $1000 \mathrm{~ms} /$ item \\
\hline$\underline{\text { Experiment 4 }}$ & $\begin{array}{c}\text { Phonologically } \\
\text { similar letters }\end{array}$ & $100,400,800 \mathrm{~ms}$ & $500 \mathrm{~ms} /$ item \\
\hline
\end{tabular}

Fig. 1 (A) Illustration of a trial within the probe-span task. Series of four red letters were presented for subsequent recall, and black probe letters were presented between the memory items, with each probe to be judged as being present in or absent from the list presented so far. At the end of the series, participants recalled the four letters in order of appearance. The

red letters in memory, and pressing the leftmost button when the black letter did not correspond to one of the red letters in memory.

The delay before probe variable was manipulated within subjects. Regardless of the delay condition, the interval between two red letters was kept constant at 2,000 ms. However, depending on the experimental condition defined by delay before probe, the delay between the offset of the red letter and the onset of the black letter was different $(100,200$, or $400 \mathrm{~ms}$ in Exp. 1; 200, 400, 600, or $800 \mathrm{~ms}$ in Exps. 2 and 3; and 100,400 , or $800 \mathrm{~ms}$ in Exp.4). Black letters were always presented for $1,000 \mathrm{~ms}$. The remaining delay between the offset of the black letter and the onset of the next red letter differed as a function of delay before probe $(900,800$, or $600 \mathrm{~ms}$, respectively, in Exp. 1; 800, 600, 400, or $200 \mathrm{ms,}$ respectively, in Exps. 2 and 3; and 900, 600, or $200 \mathrm{~ms}$, respectively, in Exp.4).

Experiments 1 and 4 included 144 trials; Experiments 2 and 3 included 96 trials. For each trial and each participant, black letters were sampled randomly from a pool of potential probes in such a way that the likelihood of receiving a positive probe was $50 \%$ at each probe position. (Positive probes consisted of any of the letters presented in the series so far.) Thus, each trial could have from zero to four positive probes. For each probe position, the pool of possible probes consisted of all the letters presented in the series so far, plus a random new letter for that series. Thus, across the entire experiment, and for each of the four probes, the black letter corresponded delay before the probe was manipulated. Here, the delay durations shown are the ones used in Experiments 2 and 3 (between 200 and $800 \mathrm{~ms}$ ). (B) Table reporting the experimental factors that could change from one experiment to another: Memory Material, Delay Before Probe, and Presentation Duration of the Memory Items

in half of the trials to one of the red memory items, and each red letter presented up to that point in the trial had equal chances of being used as a target-present probe. Importantly, in each of these four pools, every different probe type was associated equally often with each of the possible levels of delay before probe.

Before the experimental trials, participants received instructions that included a visualization of a trial. This was followed by five practice trials. Throughout the experiment, participants were asked to respond as quickly as possible to the probes, without making errors, while maintaining the four red letters in memory. They were not informed of the varying delays. Responses in the processing task were collected by button presses on a serial response box, and recall performance was scored by counting the number of letters that were correctly recalled with respect to serial order within each series $(\max =4)$. Next, an average across all series was calculated per participant.

\section{Performance-based exclusions}

In each experiment, we applied exclusion criteria as follows. First, we discarded the data of participants whose average recall score was less than one letter out of four (one, two, no, and two participants excluded in Exps. 1-4, respectively). Next, to ascertain that participants paid sufficient attention to the probe task, we excluded the data of participants who performed below 55\% correct (no, one, no, and one participant in 
Exps. 1-4, respectively). Finally, we verified participants' precise compliance with the instructions in the probe task. Because it was important that participants consider all of the red letters when judging the probe, we calculated the rate of correct responses to "not-last" probes (i.e., target-present probes that matched any but the last-presented red letter of a series) and excluded the data of participants who scored below $55 \%$ on these not-last probes (three, three, two, and three participants in Exps. 1-4, respectively). These exclusions resulted in final samples of 36 (out of 40), 54 (out of 60), 38 (out of 40), and 34 (out of 40) participants in Experiments 1 through 4, respectively.

\section{Method of analysis}

We examined the serial-position curves for the RTs collected at Probe Positions 2, 3, and 4 (following Memory Items 2, 3, and 4, respectively). Specifically, the RTs to the target-present probes were analyzed as a function of the serial position of the matching memory item. For each experiment, a separate Bayesian analysis of variance (BANOVA; Rouder, Morey, Speckman, \& Province, 2012) was run for each of the three probe positions (Probe 2, Probe 3, and Probe 4), with delay before probe (100, 200, or $400 \mathrm{~ms}$ in Exp. 1; 200, 400, 600, or $800 \mathrm{~ms}$ in Exps. 2 and 3; and 100, 400, or $800 \mathrm{~ms}$ in Exp. 4) and serial position of the matching memory item as independent within-subjects variables. The BayesFactor package for the $\mathrm{R}$ statistical analysis language was used with the default settings. For five out of the 12 ANOVAs ( 3 probe positions $\times 4$ experiments), at least one participant was missing data in one or more cells because only correct RTs were analyzed. The participants with missing data were omitted from each ANOVA, and we ran our analysis on the remaining participants. Table 1 presents the results of the analyses and reports for each analysis the number of participants included. Except for the ANOVA of the RTs collected at Probe 4 in Experiment 3, never more than one participant had missing data.

Using two variables, delay before probe and serial position, models were specified for each combination of main effects and interactions, and the BANOVA computed the Bayes factors for each of these models. We used these Bayes factors to identify the best model (i.e., the one that yielded the highest Bayes factor). As we will see, for the vast majority of our observations, the winning model included the two main effects of serial position and delay before probe, but not the interaction. We then assessed the strength of the evidence in the data against the interaction by computing a Bayes factor between the model that did not include the interaction (i.e., the main-effects-only model) and the alternative model in which the interaction term was included (i.e., the full model). The resulting Bayes factor quantified the evidence in the data against
Table 1 Evidence in the data against an interaction between serial position and delay before probe in Experiments 1 through 4

\begin{tabular}{|c|c|c|c|}
\hline & Probe 2 & Probe 3 & Probe 4 \\
\hline \multirow[t]{2}{*}{ Experiment 1} & 36 participants & 36 participants & 36 participants \\
\hline & 9.66 to 1 & 12.26 to 1 & 42.42 to 1 \\
\hline \multirow[t]{2}{*}{ Experiment 2} & 54 participants & 53 participants & 53 participants \\
\hline & 5.74 to 1 & 9.26 to 1 & 452.10 to 1 \\
\hline \multirow[t]{2}{*}{ Experiment 3} & 38 participants & 37 participants & 29 participants \\
\hline & 13.22 to 1 & 135.61 to 1 & 24.17 to 1 \\
\hline \multirow[t]{2}{*}{ Experiment 4} & 34 participants & 34 participants & 33 participants \\
\hline & 1 to 2.70 & 4.37 to 1 & 15.84 to 1 \\
\hline
\end{tabular}

Bayes factors describe the strength of the evidence for the absence of an interaction by reporting the likelihood of the main-effects-only model that does not include the interaction versus the full model in which the interaction is included

an interaction between delay before probe and serial position.

\section{Results}

\section{General performance}

As a validation of our task, as expected, participants correctly recalled several memory items at the end of the series and had high accuracy on the probes. For each experiment, recall performance and rates of correct responses to the probes by the participants in the final sets can be found in Table 2. The use of phonologically similar letters and of shorter presentation times resulted in slightly lower performance than in the other conditions, but performance remained high. There were no recognition/recall trade-offs (Supplementary Material 1).

\section{Serial-position curves}

Serial-position curves are shown for each probe delay in Fig. 2. Similar to what is typically observed in the item recognition task, we found that RTs to the probe were affected by the serial position of the matching memory item (e.g., Burrows \& Okada, 1971); across the four experiments, and

Table 2 Mean recall performance and mean probe task performance in Experiments 1-4

\begin{tabular}{lll}
\hline & Mean Items Recalled & Mean Probe Accuracy \\
\hline Experiment 1 & $3.73(.20)$ & $.94(.04)$ \\
Experiment 2 & $3.73(.22)$ & $.95(.05)$ \\
Experiment 3 & $3.28(.39)$ & $.89(.07)$ \\
Experiment 4 & $3.09(.44)$ & $.87(.06)$ \\
\hline
\end{tabular}

Standard deviations are reported in parentheses 
Experiment 1 (regular letters presented during $1000 \mathrm{~ms}$; delays from 100 to $400 \mathrm{~ms}$ )
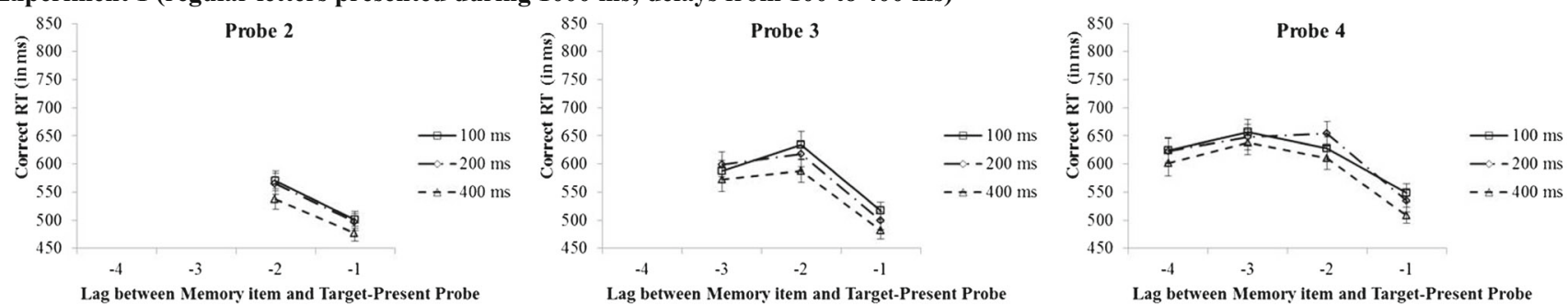

Experiment 2 (regular letters presented during $1000 \mathrm{~ms}$; delays from 200 to $800 \mathrm{~ms}$ )
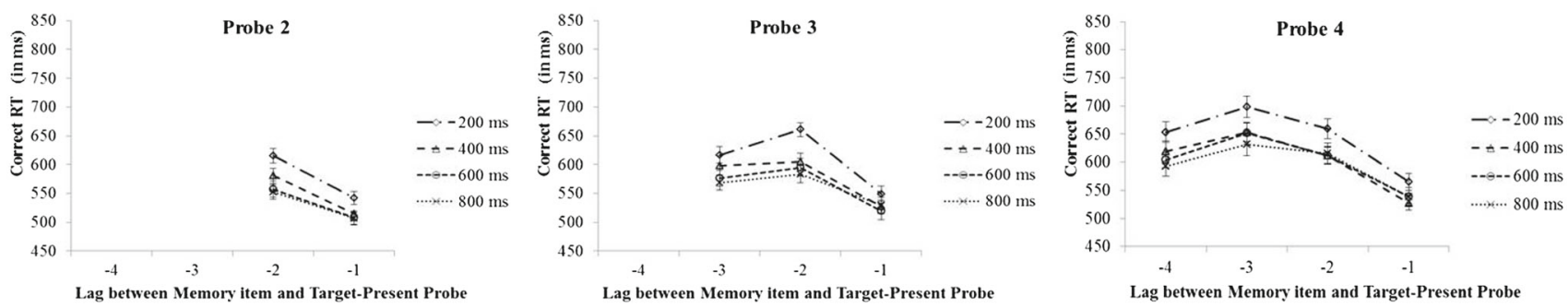

Experiment 3 (phonologically similar letters presented during $1000 \mathrm{~ms}$; delays from 200 to $800 \mathrm{~ms}$ )
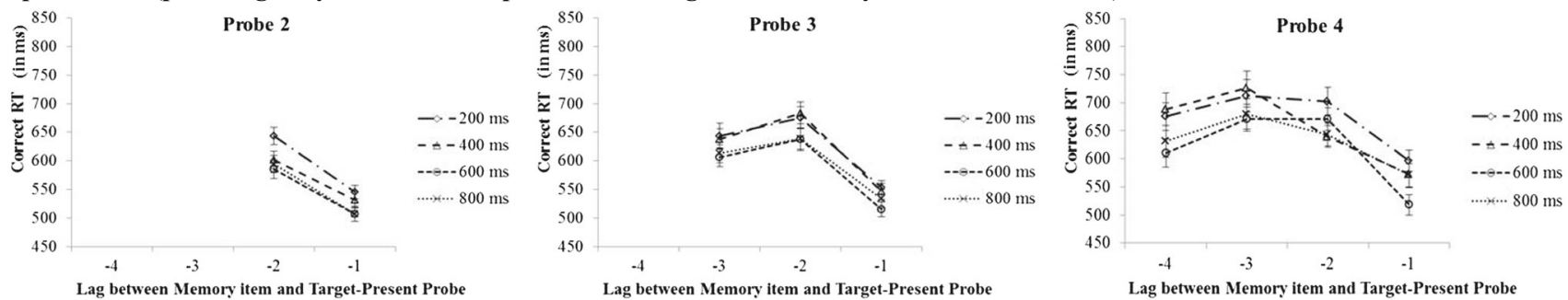

Experiment 4 (phonologically similar letters presented during $500 \mathrm{~ms}$; delays from 100 to $800 \mathrm{~ms}$ )
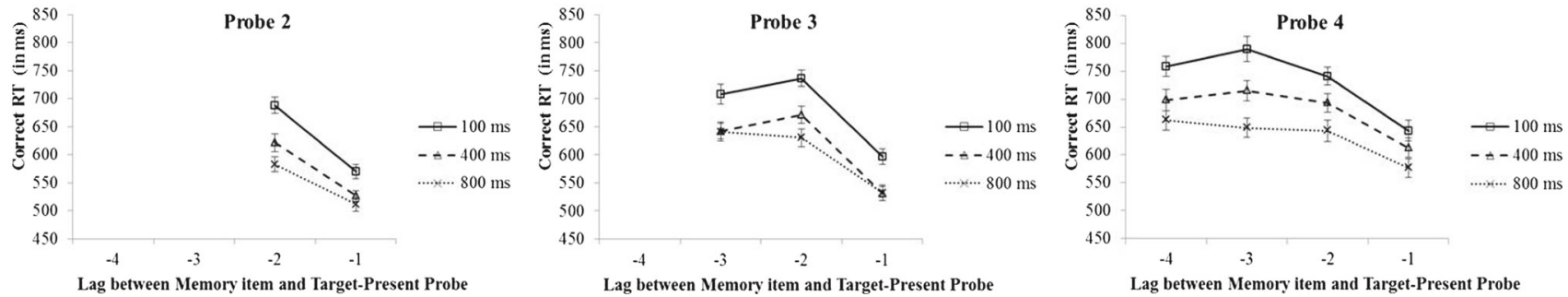

Fig. 2 Mean probe response times (RTs, in milliseconds) as a function of the serial position of the matching memory item (expressed as the lag between presentation and test; on the $x$-axis) and probe position (Probe 2, Probe 3, or Probe 4, in the left, middle, and right panels, respectively). The delays following the probe appear as the graph parameters. (Top row, Exp. 1) Regular letters were used as the memoranda and were presented for 1,000 ms; delay durations varied between 100 and $400 \mathrm{~ms}$. (Second row, Exp.2) Regular letters were used as the memoranda and were

presented for 1,000 ms; delay durations varied between 200 and $800 \mathrm{~ms}$. (Third row, Exp. 3) Phonologically similar letters were used as the memoranda and were presented for 1,000 ms; delay durations varied between 200 and 800 ms. (Fourth row, Exp.4) Phonologically similar letters were used as the memoranda and were presented for $500 \mathrm{~ms}$; delay durations varied between 100 and 800 ms. Error bars show standard errors of the means

across the different probe positions, the curves show a clear benefit for the last-presented memory item. Responses became somewhat faster after a longer delay, but it is immediately clear that one can rule out the hypothesis that the shapes of the serial-position curves changed drastically over time. Participants were fastest to respond to the last-presented memory item, and this pattern was invariant across durations of the delay before the probe. This invariance of the serial-position curves was confirmed by BANOVAs.
As can be seen in Table 1, across the three probe positions and the four experiments, the best model included the two main effects of serial position and delay before probe, but not the interaction. This model was favored over the model including the interaction by Bayes factors ranging between 9.66 and 42.42 in Experiment 1, between 5.74 and 452.10 in Experiment 2, between 13.22 and 135.61 in Experiment 3, and between 4.37 and 15.84 in Experiment 4. Only for Probe 2 in Experiment 4 was the full model with an interaction 
best, and even then only weakly, preferred over the model including only the two main effects by a factor of 2.70 . Moreover, as can be seen in the lower panel of Fig. 2, responses were still fastest to the last-presented memory item (i.e., Memory Item 2) at the longest delay. Thus, a strong pattern of invariance emerges from the ensemble of our results; RTs to the last item remained the fastest over time.

\section{Discussion}

We tested the hypothesis that attentional refreshing operates serially to maintain a set of elements. On the basis of past research, we assumed that serial refreshing could not occur much over our shortest probe delay, but would come online at longer delays. The result of this change would be a shift of the focus of attention away from dwelling on the lastpresented list item, shifting instead between items one at a time to refresh them. On the basis of the assumption that participants are faster to respond to the item that is currently in the focus of attention, as compared to any other item of the list, the serial refreshing hypothesis predicts that the item that would receive the fastest RT should change over time. Our findings contrasted sharply with this prediction: RTs to the last item were the fastest, and this pattern remained invariant over time.

Note that the experiments created optimal conditions to detect the operation of refreshing, on the basis of the assumptions put forward in the literature. After observing the unexpected invariant pattern in Experiment 1, we used delays that were more similar to the time available for refreshing in studies that have provided evidence for refreshing (Exps. 2-4). To examine whether the invariance in Experiments 1 and 2 was due to people using articulatory rehearsal rather than refreshing, we aimed at minimizing the role of articulatory rehearsal by using phonologically similar material (Exps. 3 and 4). Finally, after still not observing the expected change in the serial-position curves over time in Experiment 3, we reasoned that perhaps part of the presentation time might have been used for refreshing. To exclude this possibility, we used shorter presentation times (Exp.4), but the serial-position functions still did not change.

In what follows, we discuss what we believe are three possible accounts for the observed invariance. To account for it, one must modify either the serial-refreshing hypothesis or the hypothesis that speeded responses reflect the presence of an item in the focus of attention. Each of these accounts has potentially far-reaching implications.

According to the first account, invariant serial-position curves were observed because refreshing does not operate serially. Instead, the content of WM might be refreshed in parallel, with attention divided among the different items of the set at any point in time. To the best of our knowledge, no current WM model proposes parallel refreshing (but see Ratcliff, 1978, proposing parallel retrieval of items in WM, making parallel refreshing possible). It is worth noting that a few early studies manipulated the delay between the memory list and the single probe in the item recognition task and found serial-position curves to become somewhat flatter over time (e.g., Clifton \& Birenbaum, 1970), suggesting the possibility of serial refreshing during a retention interval following list presentation, even if not during list presentation.

According to the second account, we observed invariant serial-position curves because participants did not use refreshing in our experiments, even though we aimed to create optimal conditions to detect refreshing. At least, they might not have used refreshing during the interprobe intervals. Vergauwe and Cowan (2015) called on refreshing during probe performance to explain why a letter-probe task imposed less of a cognitive load than letter-processing tasks that did not require searching through the memoranda. However, it might be possible that in this procedure, refreshing during the probe task itself takes the place of refreshing between probes, in which case no probe delay effect on refreshing would occur. Here we did not require participants to carry out an unrelated processing task during the interletter intervals, as in most studies of refreshing (e.g., Barrouillet \& Camos, 2012), and refreshing might have taken place in the unfilled periods between such processing episodes. Alternatively, people might not refresh the content of WM unless explicitly instructed to do so (e.g., Souza et al., 2015). In that case, an alternative explanation would be needed to account for the muchreplicated cognitive-load effects on WM through which recall performance depends on the attentional demands of the secondary task (e.g., Barrouillet \& Camos, 2012). For example, an interference account does not require refreshing and would not expect attenuation of the last-item benefit to occur over time.

The first two accounts are conditional on accepting the assumption that the last-item benefit in RTs reflects the item being in the focus of attention. If one accepts that fast responses to a particular item can be used to infer the existence of a one-item focus of attention, then one must accept that we have presented strong evidence against the prevailing view that attention refreshes one element at a time to support the maintenance of a set of elements in WM; the last-item benefit should have disappeared, even if one assumes very fast refreshing.

A third account of our data, however, could involve rejecting the assumption that fast responses to a particular item can be used to infer the content of the focus of attention. An account in terms of familiarity-based recognition rather than focal attention seems unlikely, though, and is not consistent with our data (Supplementary Material 2). Most evidence for a one-item focus of attention has come from studies showing a benefit in RTs for the last-presented or - 
processed item (see Oberauer \& Hein, 2012, for a recent review). If we reject the assumption that the last-item benefit results from the last item still being in the focus of attention, then most evidence for a one-item focus of attention in WM must be discarded. It is important to note here that similar lastitem advantages have been observed when perceptual matching is prevented (e.g., McElree \& Dosher, 1989; Nee \& Jonides, 2008), excluding an account of the last-item benefit in terms of visual matching.

One might propose, though, that serial-position effects on RTs reflect different activation levels rather than items being in or out of the focus of attention (e.g., because of retroactive interference of each item with prior items). Then, serial refreshing (without severe decay) might still occur without changing the serial-position curves over time in the present experiments. Although the shapes of the serial-position curves beyond lag -1 in Fig. 2 might be consistent with the idea of different activation levels, statistical analysis does not allow firm conclusions (Supplementary Material 3).

To conclude, we observed an unexpectedly stable serialposition function that stands in contrast with what would be expected on the basis of the juxtaposition of two key assumptions about WM. The uncovered invariance puts important constraints on models of WM, attention, and human information processing; follow-up investigations should help disentangle alternative accounts.

Author note Funding for this project was provided by NIH Grant No. R01 HD-21338 to N.C., NSF Grant No. BCS-1240359 to J.N.R., and Swiss National Science Foundation Grant No. PA00P1_139604 to E.V.

\section{References}

Barrouillet, P., \& Camos, V. (2012). As time goes by: Temporal constraints in working memory. Current Directions in Psychological Science, 21, 413-419. doi:10.1177/0963721412459513

Burrows, D., \& Okada, R. (1971). Serial position effects in high-speed memory search. Perception \& Psychophysics, 10, 305-308. doi:10. 3758/BF03212831

Camos, V., Mora, G., \& Oberauer, K. (2011). Adaptive choice between articulatory rehearsal and attentional refreshing in verbal working memory. Memory \& Cognition, 39, 231-244. doi:10.3758/s13421010-0011-x

Camos, V., \& Portrat, P. (2015). The impact of cognitive load on delayed recall. Psychonomic Bulletin \& Review, 22, 1029-1034. doi:10. 3758/s13423-014-0772-5

Clifton, C., \& Birenbaum, S. (1970). Effects of serial position and delay of probe in a memory scan task. Journal of Experimental Psychology, 86, 69-76. doi:10.1037/h0029980
Cowan, N. (1995). Attention and memory: An integrated framework. New York, NY: Oxford University Press.

Cowan, N. (2011). The focus of attention as observed in visual working memory tasks: Making sense of competing claims. Neuropsychologia, 49, 1401-1406. doi:10.1016/j. neuropsychologia.2011.01.035

Higgins, J. A., \& Johnson, M. K. (2009). The consequence of refreshing for access to nonselected items in young and older adults. Memory \& Cognition, 37, 164-174. doi:10.3758/MC.37.2.164

Loaiza, V. M., Duperreault, K. A., Rhodes, M. G., \& McCabe, D. P. (2015). Long-term semantic representations moderate the effect of attentional refreshing on episodic memory. Psychonomic Bulletin \& Review, 22, 274-280. doi:10.3758/s13423-014-0673-7

McCabe, D. P. (2008). The role of covert retrieval in working memory span tasks: Evidence from delayed recall tests. Journal of Memory and Language, 58, 480-494. doi:10.1016/j.jml.2007.04.004

McElree, B. (2006). Accessing recent events. In B. H. Ross (Ed.), The psychology of learning and motivation (Vol. 46, pp. 155-200). San Diego, CA: Academic Press. doi:10.1016/S0079-7421(06)46005-9

McElree, B., \& Dosher, B. A. (1989). Serial position and set size in shortterm memory: The time course of recognition. Journal of Experimental Psychology: General, 118, 346-373. doi:10.1037/ 0096-3445.118.4.346

Nee, D. E., \& Jonides, J. (2008). Neural correlates of access to short-term memory. Proceedings of the National Academy of Sciences, 15, 14228-14233. doi:10.1073/pnas.0802081105

Nee, D. E., \& Jonides, J. (2013). Trisecting representational states in short-term memory. Frontiers in Human Neuroscience, 7, 796. doi: $10.3389 /$ fnhum.2013.00796

Oberauer, K., \& Hein, L. (2012). Attention to information in working memory. Current Directions in Psychological Science, 21, 164169. doi:10.1177/0963721412444727

Oberauer, K., \& Lewandowsky, S. (2011). Modeling working memory: A computational implementation of the Time-Based Resource-Sharing theory. Psychonomic Bulletin \& Review, 18, 10-45. doi:10.3758/ s13423-010-0020-6

Oztekin, I., Dvachi, L., \& McElree, B. (2010). Are representations in working memory distinct from representations in long-term memory? Psychological Science, 21, 1123-1133.

Ratcliff, R. (1978). A theory of memory retrieval. Psychological Review, 85, 59-108. doi:10.1037/0033-295X.85.2.59

Rouder, J. N., Morey, R. D., Speckman, P. L., \& Province, J. M. (2012). Default Bayes factors for ANOVA designs. Journal of Mathematical Psychology, 56, 356-374. doi:10.1016/j.jmp.2012. 08.001

Souza, A. S., Rerko, L., \& Oberauer, K. (2015). Refreshing memory traces: Thinking of an item improves retrieval from visual working memory. Annals of the New York Academy of Sciences, 1339, 2031. doi:10.1111/nyas. 12603

Vergauwe, E., Camos, V., \& Barrouillet, P. (2014). The effect of storage on processing: How is information maintained in working memory? Journal of Experimental Psychology: Learning, Memory, and Cognition, 40, 1072-1095. doi:10.1037/a0035779

Vergauwe, E., \& Cowan, N. (2015). Attending to items in working memory: Evidence that refreshing and memory search are closely related. Psychonomic Bulletin \& Review, 22, 1001-1006. doi:10.3758/ s13423-014-0755-6 